Die Rolle von Massenmedien für die Ökologisierung von Unternehmensstrategien

\section{Protagonisten, Plattformen, Partner}

\author{
Den Massenmedien kommt im Spannungsfeld von Ökologie und Wettbewerbs- \\ fähigkeit eine zentrale Bedeutung zu. Sie verschaffen Zugang zur gesell- \\ schaftspolitischen Öffentlichkeit, in der über die Akzeptanz und Legitimität von \\ Unternehmensstrategien entschieden wird. Öko-Strategien erfordern deshalb \\ eine Kommunikationspolitik, die alle Facetten der Medienarbeit beherrscht.
}

$\mathrm{D}$

Von Ansgar Zerfaß er Schlüssel zum Erfolg im globalen Wettbewerb sind innovative Produkte und Dienstleistungen, die vom Markt nachgefragt werden. Auch umweltbewußte Produktion und ökologische Produkte müssen sich in der Marktwirtschaft letztlich rechnen. Unternehmen müssen jedoch immer auch bemüht sein, so zu agieren, daß die Verfolgung von Marktzielen nicht gegen rechtliche oder moralische Normen verstößt. Wenn diese doppelte Anforderung nicht erfüllt wird, droht einerseits der ökonomische Niedergang, andererseits der (schleichende) Entzug der „license to operate“ durch gesetzliche Auflagen, öffentliche Kritik und nachhaltigen Glaubwürdigkeitsverlust. Diese praktische Erfahrung kann theoretisch nachvollzogen werden, wenn man Unternehmen als soziale Institutionen betrachtet, die gleichzeitig in verschiedenen gesellschaftlichen Handlungsfeldern (Markt, Politik, Öffentlichkeit) agieren und dabei mit den Ansprüchen verschiedenster Bezugsgruppen konfrontiert werden.

Bei der Positionierung in Markt und Gesellschaft kommt der Kommunikationspolitik eine immer wichtigere Bedeutung zu. Umweltkommunikation als Bestandteil einer integrierten Unternehmenskommunikation erfüllt dabei mehrere Aufgaben: Einerseits unterstützt sie die Entwicklung, Umsetzung und Vermarktung ökologischer Produkte durch die Einbindung entsprechender Themen bzw. Argumente in die Mitarbeiter- und Marktkommunikation. Andererseits - und darum geht es uns im folgenden - ist die Öko-PR der entscheidende Ansatzpunkt für die Teilnahme am gesellschaftlichen Diskurs über Umweltfragen.

Die ökologische Diskussion wird in verschiedenen Arenen gefïhrt, beispielsweise im privaten Bereich, in der Fachöffentlichkeit der Umweltforscher und in der verbandsinternen öffentlichkeit von Umweltinitiativen. Den entscheidenden Einfluß auf Markterfolg und Legitimität hat jedoch die gesellschaftspolitische Öffentlichkeit, jene laiensprachlich verfaßte Kommunikationsarena, die als Knotenpunkt im Geflecht verschiedener Öffentlichkeiten gilt und grundsätzlich allen Mitgliedern der Gesellschaft offensteh. In dieser Arena entsteht öffentliche Meinung im Sinne der Weltbilder und Wertungen, die sich im Verlauf der Diskussion durchgesetzt haben und somit eine „herrschende“ Meinung darstellen. Diese Mehrheitsmeinung hat unmittelbare Auswirkungen auf die Anforderungen, die an die ökologische Performance von Unternehmen gestellt werden, und auf die Einstellungen, die man konkreten öko-Produkten entgegenbringt.

In modernen Gesellschaften wird die Funktionsfähigkeit der gesellschaftspolitischen öffentlichkeit maßgeblich durch Massenmedien wie Presse, Rundfunk und offene Datennetze sichergestellt. Die Kommunikation folgt dabei zwangsläufig eigenen Regeln: Wenigen Sprechern und ebenso wenigen Mittlern (Reporter, Journalisten) stehen viele Rezipienten gegenüber, die sich für konkrete Medienprodukte (Zeitungen, Sendungen) interessieren und dadurch ein sehr heterogenes Publikum bilden. Was auf die Agenda der Massenmedien kommt und wie einzelne Themen dort bewertet werden, wird von journalistischen Arbeitsroutinen, dem Nachrichtenwert einzelner Meldungen und nicht zuletzt vom ökonomischen Interesse der Medienunternehmen selbst beeinflußt.

Die Massenmedien haben die Debatte über Umweltthemen in den 70er Jahren öffentlich gemacht und seitdem maßgeblich vorangetrieben. Verschiedene Untersuchungen zeigen aber, daß durch die massenmediale Darstellung nicht zwangsläufig differenzierte Problemsichten vermittelt oder rationale Entscheidungen befördert werden (1). Massenmedien stellen ökologische Fragen überwiegend ereignisbezogen, skandalisierend, emotional und ohne Bezug auf einheitliche Bewertungsstandards dar. Komplexe Sach- verhalte wie das Zusammenspiel von Ökologie und Unternehmensführung werden auf einfache Schemata reduziert - Unternehmen sind potentielle Umweltsünder, Umweltaktivisten selbstlose Vertreter des Gemeinwohls und Produkte mit Öko-Appeal stets die bessere Wahl. Die Umweltkommunikation muß sich diese Rahmenbedingungen vergegenwärtigen und Strategien für eine aktive Öko-PR entwerfen.

\section{- Aktionsfelder von Öko-PR}

Das generelle Ziel der Öko-PR ist die Interessenklärung und Handlungskoordination mit Bezugsgruppen im gesellschaftspolitischen Umfeld der Unternehmung (2). Besondere Bedeutung haben dabei ökologische Anspruchsgruppen, die sich besonders mit Umweltthemen identifizieren und deshalb die Diskussion deutlich stärker als Anwohner, Politiker, Wissenschaftler usw. selbst in Gang bringen und prägen. Eine erfolgreiche Öko-PR setzt immer auf mehrere Kommunikationswege (vgl. Abb. 1):

- Gut steuerbar ist die direkte Öffentlichkeitsarbeit, bei der man sich mit Umweltberichten, Infobroschüren, Umwelt-Events und Websites im Internet direkt an die Bezugsgruppen wendet. Obwohl dabei der Aufbau eines Öko-Images im Vordergrund steht, bieten sich doch auch Ansatzpunkte für Rückfragen und damit für den Einstieg in zweiseitige, dialogische Kommunikationsprozesse.

- Besonders leistungsfähig, aber auch mit hohen Kosten und Risiken verbunden ist die Dialogkommunikation im Sinne „runder Tische" und Konsultationsverfahren mit ökologischen Anspruchsgruppen (3). Solche Unternehmensdialoge, die der Sensibilisierung für ökologische Themen, der Diskussion von Risiken und der Regulierung von Konflikten dienen können, werden beispielsweise in der Chemischen Industrie mit Erfolg durchgeführt.

- Die Massenmedien und insbesondere Journalisten als handelnde Akteure spielen eine dreifache Rolle: Sie sind zugleich Protagonisten, Plattform und Partner für die öffentliche Diskussion über ökologische Unternehmensstrategien.

\section{- Protagonisten für umweltbewußtes Handeln}

Massenmedien bringen Themen auf die öffentliche Agenda. Sie prägen unser Bild der Welt und nehmen maßgeblich darauf Einfluß, wie Problemlagen definiert und welche Lösungswege diskutiert werden. Ein investigativer Journalis- 
mus weist immer wieder aktiv auf neue Aspekte im Verhältnis von Unternehmen und natürlicher Umwelt hin. In diesem Fall werden die Massenmedien zu Protagonisten des umweltbewußten Handelns. Als Vorkämpfer für bestimmte Sichtweisen und Wertungen tragen sie dazu bei, daß sich die Anforderungen an unternehmerisches Handeln ändern. Aufgrund der oben skizzierten Funktionsweise des Mediensystems ist keineswegs sicher, daß damit stets zielfiihrende Diskussionsprozesse angestoßen und rationale Lösungen befördert werden. Die öffentliche Thematisierung kann dazu führen, daß bestimmte Wettbewerbsstrategien und Handlungsweisen erschwert oder gar durch den Aufbau eines Negativimages unmöglich gemacht werden. Andererseits können Unternehmen die Popularität ökologischer Themen nutzen, um ein positives Image aufzubauen und bestimmte ProduktMarkt-Konzepte („Dreiliterauto“) mit Erfolg durchzusetzen.

\section{Plattformen für ökologische Kommunikation}

Massenmedien sind zweitens die zentrale Plattform für öffentliche Auseinandersetzungen und Meinungsäußerungen über Umweltfragen. Dabei sind zwei Fälle zu unterscheiden (vgl. Abb. 1): Einerseits wird die Kommunikation zwischen Unternehmen und ökologischen Anspruchsgruppen zum Teil bewußt über die Massenmedien geführt (Öffentliche Koтmunikation). Beispielsweise inszenieren Greenpeace und andere Umweltinitiativen immer wieder medienwirksame Aktionen, um Forderungen an die Industrie zu stellen und diesen gleich den notwendigen Nachdruck zu verleihen. Unternehmen und Verbände antworten dann ebenso öffentlich in Pressekonferenzen und TV-Statements. Die Diskussion wird schnell zur Scheindebatte, die sich an journalistischen Aufmerksamkeitsregeln orientiert und erst dann wirkliche Lösungen verspricht, wenn entweder eine Seite den Kampf um die öffentliche Meinung gewinnt („Brent Spar“) oder wenn die Auseinandersetzung in den nicht-öffentlichen Raum der Dialogkommunikation und direkter Gespräche verlagert wird. Andererseits gehen sowohl Unternehmen als auch ökologische Anspruchsgruppen direkt auf die Massenmedien zu, um bestimmte Themen und Interpretationen durchzusetzen (Medienarbeit). Empirische Untersuchungen zeigen, daß der Einfluß der Pressearbeit auf die Darstellung ökologischer

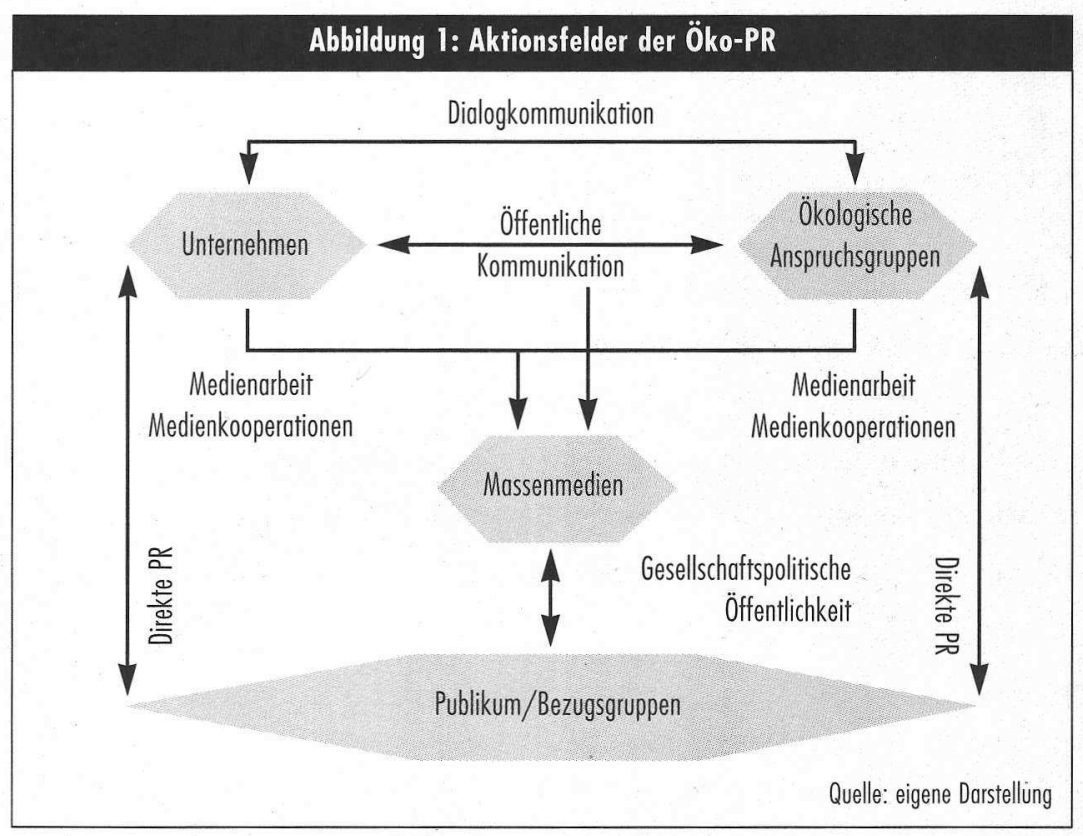

Sachverhalte sehr groß ist. Im Umweltbereich, der in den seltensten Fällen von fachkundigen Journalisten oder Ressorts bearbeitet wird, sind die Massenmedien zwangsläufig auf den Input der Hauptakteure angewiesen. Öko-Initiativen genießen dabei häufig einen Vertrauensvorsprung gegenüber Unternehmen und Wirtschaftsverbänden, deren Argumente gegen die plakativen und emotionalen Aussagen von Greenpeace \& Co. manchmal kein Gehör finden. Dieses Dilemma ist eine Folge der Wagenburgmentalität, mit der weite Teile der Industrie in den 70er und 80er Jahren auf ökologische Forderungen reagiert haben. Die Wiedergewinnung von Vertrauen und Legitimität durch offene Kommunikation ist - wie das Beispiel der Chemischen Industrie zeigt - ein langwieriges Geschäft.

\section{Partner für Öko-Strategien}

Massenmedien sind drittens ein wichtiger Partner für die Umsetzung ökologischer Wettbewerbsstrategien. Das Thema Ökologie steht auf der gesellschaftspolitischen Agenda nicht mehr ganz oben, ist aber längst in allen Köpfen verankert und somit zum Dauerbrenner geworden. Deshalb haben die Massenmedien, die als Wirtschaftsorganisationen jenseits ihres Selbstverständnisses als neutrale Informationsmittler und vierte Macht im Staat immer an die Auflage bzw. Reichweite denken müssen, ein latentes Eigeninteresse an der Besetzung ökologischer Themen. Aus dieser Konstellation heraus entstehen immer wieder mehr oder weniger offene
Medienkooperationen: Partnerschaften zwischen Unternehmen und Journalisten, die über innovative Produktionsweisen und Öko-Produkte berichten, aber auch Allianzen zwischen Massenmedien und ökologischen Anspruchsgruppen, die - beispielsweise im Zuge von Produkttests - bestimmte Umweltaspekte thematisieren und mit positiven oder negativen Wertungen verbinden.

Nicht zuletzt deshalb muß eine strategisch geplante Umweltkommunikation zum integralen Bestandteil der Unternehmensstrategie werden.

\section{Anmerkungen}

(1) Vgl. Dernbach, B.: Public Relations für Abfall. Ökologie als Thema öffentlicher Kommunikation, Opladen/Wiesbaden 1998.

(2) Vgl. Fichter, K.: Umweltkommunikation und Wettbewerbsfähigkeit, Marburg 1998, und

Zerfaß, A.: Unternehmensführung und Öffentlichkeitsarbeit, Opladen 1996.

(3) Bentele, G./ H. Steinmann, H./ A. Zerfaß (Hrsg.): Dialogorientierte Unternehmenskommunikation. Berlin 1996.

\section{Der Autor}

Dr. Ansgar Zerfaß leitet den Bereich Kommunikation/Öffentlichkeitsarbeit bei der MFG Medien- und Filmgesellschaft Baden-Württemberg, Stutigart, und ist Herausgeber von www.pr-guide.de, dem OnlineDienst der deutschen PR-Branche.

Kontakt: Obere Wiesenstr. 13, 73760 Ostfildern, E-Mail: mail@zerfass.de 
(c) 20I0 Authors; licensee IÖW and oekom verlag. This is an article distributed under the terms of the Creative Commons Attribution Non-Commercial No Derivates License (http://creativecommons.org/licenses/by-nc-nd/3.o/), which permits unrestricted use, distribution, and reproduction in any medium, provided the original work is properly cited. 